\title{
Adaptive Traffic Light Detection using Color Spaces
}

\author{
Aradhana Verma \\ Research Scholar \\ Noida institute of Engineering \\ and Technology, Greater \\ Noida, India
}

\author{
C.S. Yadav, PhD \\ Professor Dept. of Computer \\ Science \\ Noida institute of Engineering \\ and Technology, Greater \\ Noida, India
}

\author{
Pradeep Kumar \\ Assistant Professor Dept. of \\ Computer Science \\ Noida institute of Engineering \\ and Technology, Greater \\ Noida, India
}

\begin{abstract}
This paper presents a color space based algorithm for traffic signal light detection for the modules used in Advanced Driver Assistance Systems (ADAS). The autonomous vehicle has been a topic for the discussions among the computer science engineers for several years. Traffic Signal Light Detection algorithm is based on color space theory which efficiently detects the color of traffic light during day time as well as night time i.e. weather invariant..
\end{abstract}

\section{General Terms}

ADAS, Color Space, Traffic Light

\section{Keywords}

ADAS, Autonomous Vehicle Theory, TSLD, Color Space

\section{INTRODUCTION}

Last few decades the automobile industry has grown very rapid and the number of cars producing by it is even more. This brought about increase in traffic and its rules with traffic signals, signs and police. Driving turned into a complex job and the amount of accidents increasing consistently year by year. About 1 crore individuals get affected by accidents around the globe every year and twenty to thirty lakh of these people are harmed severely. Both automobile manufactures and mainstream researchers have helped the improvement of distinctive sorts of security frameworks so as to enhance traffic safety. At first, enhancements comprised of basic components like seat belts, however then more intricate devices, for example, electronically monitored slowing mechanisms, electronic adjustment projects, and airbags, were produced. In the course of the most recent decade, research has moved to more advanced situations where a system can predict the chances of accidents and give the information to the driver for alertness. These are known as Advanced driving assistance systems (ADASs), since they aid the driver in checking chances of accidents, give motions in potentially safer driving circumstances, and execute balancing measures. A few illustrations are the versatile journey control, which keeps up a safe gap in between lane and the vehicle cautioning that does when the vehicle is determined out of a path coincidentally. To decrease number of accidents, earlier automobile producers enhanced their vehicles with the presentation of better brakes and tires. It reduced the occurrences of accidents reliably for an extent, but it did not deal with the things which causes mainly for accidents. For that reason automobile industry put forward their research on other safety measuring elements such as air bags, automatic braking system etc. which is termed as Advanced Driving Assistance Systems (ADAS), with this improvements they moved further for Night Vision systems which indeed works on mainly image processing principles with the assistance of a camera and processing unit.
Advanced Driver Assistance Systems, or ADAS, are systems designed to help the driver in the driving process. These systems are designed to be embedded in cars in order to give hints to the driver and at the same time be able to correct some driving errors trying to avoid accidents without being intrusive to the driver. There are many examples of these systems: adaptive curse control (ACC), lane departure warning system (LDWS), collision avoidance system (CAS), vehicle detection system, traffic sign and light detection system, blind spot detection system (BSD), etc.

\section{PROPOSED WORK}

\subsection{Traffic Signal Light Detection}

Reliable traffic light recognition has been one of the main challenging problems since the introduction of autonomous vehicles. Traffic light recognition has become really important for Driver Assistance Systems (DAS). It provides essential information to the driver on intersections and crosswalks and it can reduce the number of crashes for missed traffic light due to distraction. A wearable device could be designed for the visually impaired and the blind to help them safely cross streets.

\subsection{For Day Light Detection}

The region of interest is selected for the removal of areas which cannot contain the traffic light. The lower part of the image can never contain the traffic light in the image. The candidates for the traffic light is extracted and checked for its probable size in pixels. Very big and too small candidates are rejected. For the remaining candidates we find the $\mathrm{YCbCr}$ image and check if mean $\mathrm{Cr}$ value of the candidate is more than a threshold (100) and more than its nearby area. If the candidate passes the test it is taken ahead else it is dropped. For detecting green color in the day we use the HSV color space. Thresholding the HSV color image gives us the base image for green color detection. Value frame below .4 is suppressed to 0 and value frame between 0.4 and 0.5 is also suppressed to 0 if the saturation frame is less than 0.4. In the base image region of interest is extracted. The candidates in this region of interest are checked for their size. The remaining candidates are passed through a test which checks if the hue and value of the candidate falls in the green region or not. Also as an additional check mean $\mathrm{Cr}$ frame value of the candidate is checked $(<120)$ to validate it. The detected red and green candidates are checked for symmetry i.e. do the detected ones lie in the same line. The out of line candidates are dropped. This gives us the final traffic light.

\subsection{For Night Time Detection}

For the night time we us the grayscale frame of the colored image. The image is converted into black and white image and used as our base image for detection. Further the region of interest is selected. Candidates in the region of interest are tested for size and proportion. The candidates not applying the 
defined size and proportions are dropped. For the remaining candidates we find the mean red, green and blue value inside the candidate and also for the local neighborhood of the candidate. For detecting red color we check if red mean inside the candidate is high, the difference between mean red and green color is more (>30) and the mean red color outside the candidate in the local neighborhood is less than it. For detecting the green color we check if the mean green value of the candidate is high, the difference between mean red and green value is more and the mean green color outside the candidate in the local neighborhood is less than it. Further the detected candidates are checked for alignment and proportion. The final detected candidate is the color of the traffic light.

\section{RESULTS}

\subsection{Traffic Signal Light Detection}

Fig. 1 (a) and 1 (b) depicts the results of traffic signal light detection during the day and night time. The algorithm was tested in different environment conditions and was found to be accurate under all the conditions.

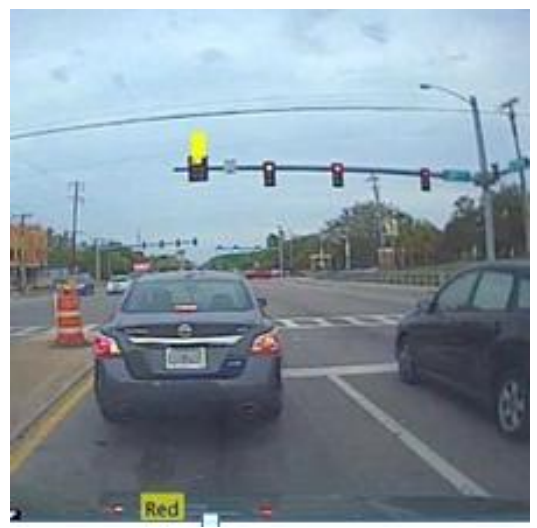

Fig1(a): Daytime Detection

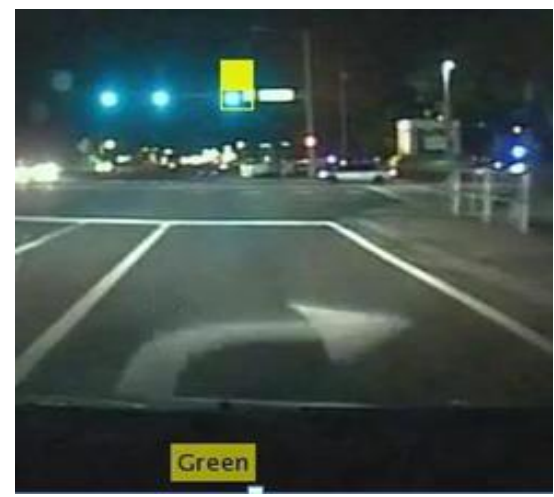

Fig 1(b): Night Time Detection

At night green color can be found near blue lights and thus can create problem but the proper selection of region of interest and color space helps us detect the light.

\section{CONCLUSION}

As a contribution to the autonomous vehicles research the a module of the advanced driver assistance system are presented in this paper i.e. traffic light detection. The results of the paper prove the accuracy of the detection algorithms for traffic signal lights road during day and night. Color space based algorithm has been implemented for the traffic signal light detection. It is applicable in autonomous vehicle concept.

\section{REFERENCES}

[1] V. Anh, A. Ramanandan, C. Anning, J. A. Farrell, and M. Barth, "Real-Time Computer Vision/DGPS-Aided Inertial Navigation System for Lane-Level Vehicle Navigation," Intelligent Transportation Systems, IEEE Transactions on, vol. 13, pp. 899-913, 2012.

[2] Z. Cai, M. Gu, and Y. Li, "Real-time arrow traffic light recognition system for intelligent vehicle," Las Vegas, NV, 2012, pp. 848-854.

[3] M. Diaz-Cabrera, P. Cerri, and J. Sanchez-Medina, "Suspended traffic lights detection and distance estimation using color features," Anchorage, AK, 2012, pp. 1315-1320.

[4] J. Gong, Y. Jiang, G. Xiong, C. Guan, G. Tao, and H. Chen, "The recognition and tracking of traffic lights based on color segmentation and CAMSHIFT for intelligent vehicles," La Jolla, CA, 2010, pp. 431-435.

[5] M. Omachi and S. Omachi, "Detection of traffic light using structural information," Beijing, 2010, pp. 809812 .

[6] Y. Shen, U. Ozguner, K. Redmill, and J. Liu, "A robust video based traffic light detection algorithm for intelligent vehicles," Xi'an, 2009, pp. 521-526.

[7] H. Tae-Hyun, J. In-Hak, and C. Seong-Ik, "Detection of traffic lights for vision-based car navigation system," vol. 4319 LNCS, ed. Hsinchu, 2006, pp. 682-691.

[8] BoWu and Ram Nevatia, "Detection and Segmentation of Multiple, Partially Occluded Objects by Grouping, Merging, Assigning Part Detection Responses,' International Journal of Computer Vision, vo. 82, pp. 185-204, 2009.

[9] Wen-Chang Cheng and Ding-MaoJhan, "A selfconstructing cascade classifier with AdaBoost and SVM for pedestrian detection," Engineering Applications of Artificial Intelligence, vol. 26, pp. 1016-1028, 2013.

[10] Rodrigo Verschae, Javier Ruiz-del-Solar and Mauricio Correa, "A unified learning framework for object detection and classification using nested cascades of boosted classifiers," Machine Vision and Applications, vol. 19, pp. 85-103, 2008.

[11] Joo Kooi Tan, Kazuki Inumaru, Seiji Ishikawa, and Takashi Morie, "Automatic detection of pedestrians from stereo camera images," Artif Life Robotics, vol. 15, pp. 459-463, 2010.

[12] Gianluca Antonini, Santiago Venegas Martinez, Michel Bierlaire, and Jean Philippe Thiran, "Behavioral Priors for Detection and Tracking of Pedestrians in Video Sequences," International Journal of Computer Vision, vol. 69(2), pp. 159-180, 2006.

[13] Azra Habibovic, Emma Tivesten, Nobuyuki Uchida, Jonas Bärgman, and Mikael Ljung Austa, "Driver behavior in car-to-pedestrian incidents: an application of the Driving Reliability and Error Analysis Method (DREAM)," Accident Analysis and Prevention, vol. 50, pp. 554- 565, 2013.

[14] Yu-Ting Chen and Chu-Song Chen, "Fast Human Detection Using a Novel Boosted Cascading Structure With Meta Stages," IEEE Transactions on Image Processing, vol. 17 (8), pp. 1452-1464, 2008. 